\title{
New sequential algorithm using Mac-2 binding protein glycosylation isomer to detect advanced carotid artery atherosclerosis
}

\author{
Huiyul Park ${ }^{1}$, Dae Won Jun ${ }^{2}$, Hoon-Ki Park ${ }^{1}$, Kye-Yeung Park ${ }^{1}$, Hwan-Sik Hwang ${ }^{1}$ \\ ${ }^{1}$ Department of Family Medicine, Hanyang University College of Medicine, Seoul, Korea; ${ }^{2}$ Department of Internal Medicine, Hanyang University \\ College of Medicine, Seoul, Korea \\ Contributions: (I) Conception and design: DW Jun; (II) Administrative support: DW Jun; (III) Provision of study materials or patients: HK Park, HS \\ Hwang; (IV) Collection and assembly of data: H Park; (V) Data analysis and interpretation: KY Park; (VI) Manuscript writing: All authors; (VII) \\ Final approval of manuscript: All authors. \\ Correspondence to: Dr. Dae Won Jun. Department of Internal Medicine, Hanyang University College of Medicine, 222 Wangsimni-ro, Seongdong- \\ gu, Seoul, 133-791, Korea. Email: noshin@hanyang.ac.kr; Hoon-Ki Park. Department of Family Medicine, Hanyang University College of \\ Medicine, 222 Wangsimni-ro, Seongdong-gu, Seoul, 133-791, Korea. Email: hoonkp@hanyang.ac.kr.
}

Background: Although carotid artery sonography is widely performed, most guidelines do not recommend this procedure in the general population. Appropriate indications and effective algorithms are needed to detect advanced carotid artery atherosclerosis in a community setting.

Methods: This study was designed as cross-sectional study. Adult subjects ( $n=228)$ who underwent a health check-up at our healthcare centre were included in the final analysis. Mac-2 binding protein glycosylation isomer (M2BPGi) quantification was based on a lectin antibody sandwich immunoassay. Subclinical atherosclerosis was diagnosed by carotid ultrasonography.

Results: The prevalence of subclinical atherosclerosis and advanced atherosclerosis was 37.2\% (85/228) and $11.8 \%$ (27/228), respectively, in a community-based setting. Serum M2BPGi level was significantly higher in subjects with calcified plaque (0.6317) and luminal stenosis (0.6373) than in control groups $(0.4913$, all $\mathrm{P}<0.05)$. Pearson correlation analysis between M2BPGi and atherosclerotic cardiovascular disease (ASCVD) risk index $(\mathrm{R}=0.410, \mathrm{P}<0.001)$ showed a positive relationship. The AUROC of serum M2BPGi for identifying calcified plaque or luminal stenosis was 0.679 . The sequential algorithm using ASCVD and M2BPGi showed good negative predictive value (NPV) (93.6\%) and reasonable positive predictive value (PPV) (53.8\%) for identifying calcified plaque or luminal stenosis. When the sequential algorithm was used as an indicator for carotid ultrasonography, 35.0\% (14/40) of subjects with intermediate-risk by ASCVD $(\geq 7.5 \%)$ could avoid unnecessary carotid ultrasonography.

Conclusions: The sequential algorithm using ASCVD $(\geq 7.5)$ and M2BPGi $(\geq 0.525)$ provided reasonable indication for carotid artery sonography in a community-based setting.

Keywords: Subclinical atherosclerosis; Mac-2 binding protein glycosylation isomer (M2BPGi); atherosclerotic cardiovascular disease risk (ASCVD)

Submitted Oct 31, 2020. Accepted for publication Jan 08, 2021.

doi: 10.21037/atm-20-7219

View this article at: http://dx.doi.org/10.21037/atm-20-7219 


\section{Introduction}

Atherosclerosis is a chronic and progressive vascular disease in which the inside of an artery narrows due to the build-up of plaque. Its main clinical manifestations are ischaemic heart disease, ischaemic stroke and peripheral arterial disease and it is recognized as a leading cause of vascular disease worldwide $(1,2)$. Because most patients with atherosclerosis have no symptoms, conventional cardiovascular risk scoring systems underestimate or misclassify the risk of cardiovascular disease and there are lots of patients with a high risk of cardiovascular disease in the population with subclinical atherosclerosis. An appropriate strategy is needed for the screening, identification and healthcare coordination of patients with subclinical atherosclerosis in the general population (3-5).

Generally, most guidelines recommend to use risk assessment methods to check major cardiovascular risk factors, such as older age, male sex, hypertension, smoking, hypercholesterolemia, and diabetes or to use the cardiovascular risk scoring systems such as atherosclerotic cardiovascular disease (ASCVD) risk estimator to screen subjects with high risk for cardiovascular disease in the general population $(6,7)$. There are not specific biomarkers to be used for screening subjects with high risk for cardiovascular disease in the general population. Although there are discrepancies between the guidelines on the role of carotid artery sonography in asymptomatic populations, most do not recommend carotid artery sonography screening of advanced carotid artery disease in the general population. The US Preventive Services Task Force does not recommend carotid screening in adult patients without a history of stroke (6). Other guidelines recommend carotid artery imaging for limited indications in the general population (e.g., carotid bruit or multiple vascular risk factors) $(8,9)$. However, most carotid artery sonography is performed widely without appropriate indications or prior risk assessment in a real practice. Therefore, an appropriate algorithm is needed to identify high-risk subjects for carotid ultrasound in a community-based setting.

An ASCVD risk estimator is designed to estimate cardiovascular events within 10 years. If the ASCVD risk score is 7.5 or higher, it is classified as an intermediaterisk with $7.5 \%$ or higher cardiovascular incidents within 10 years, and statin use is recommended. However, there is some discordance between the 10-year ASCVD risk scores calculated from clinical and blood assessment and carotid artery imaging findings (10).
Mac-2 binding protein glycosylation isomer (M2BPGi) has been studied as a useful serum marker for evaluating liver fibrosis in various chronic liver diseases $(11,12)$ and malignant tumours $(11,13)$. Although more studies are needed, M2BPGi functions at a molecular level as a ligand for E-selectin, which plays an important role in cell adhesion (14,15), as a proinflammatory cytokine (11) and the suppressive effect of adiponectin (16) are revealed. Sugiura et al. firstly reported that M2BPGi has a positive relationship with the severity of cardiovascular risk, the augmentation index, which is an indirect indicator of vascular stiffness, and diacron reactive oxygen metabolites (d-ROM), which is a metabolite of oxidative stress, in crosssectional (17) and longitudinal (18) studies. Sugiura et al. showed that M2BPGi has potential as a biomarker for atherosclerosis diagnosis. However, an indirect method was used as a diagnostic tool for atherosclerosis, and diagnostic performance was not evaluated in their studies.

Therefore, we evaluated the association between serum M2BPGi and the severity of subclinical atherosclerosis diagnosed by carotid ultrasonography and assessed the diagnostic performance of serum M2BPGi for identifying the different stages of subclinical atherosclerosis. Finally, we proposed a new sequential algorithm; ASCVD risk evaluation was used before serum M2BPGi to identify the indication for carotid ultrasound and to detect advanced atherosclerosis in a community-based setting. We present the following article in accordance with the STROBE reporting checklist (available at http://dx.doi.org/10.21037/ atm-20-7219).

\section{Methods}

\section{Study design}

This was designed as a cross-sectional study. The medical records of visitors attending a health check-up at Hanyang International University Hospital were collected for analysis. Only serum M2BPGi was measured additionally by using remnant blood samples. The authors are accountable for all aspects of the work in ensuring that questions related to the accuracy or integrity of any part of the work are appropriately investigated and resolved. The study was conducted in accordance with the Declaration of Helsinki (as revised in 2013). The Institutional Review Board (IRB) of Hanyang University Medical Centre approved this study protocol (IRB No. HYUH 2020-01-035). The protocol was also registered at the Clinical Research Information Service 


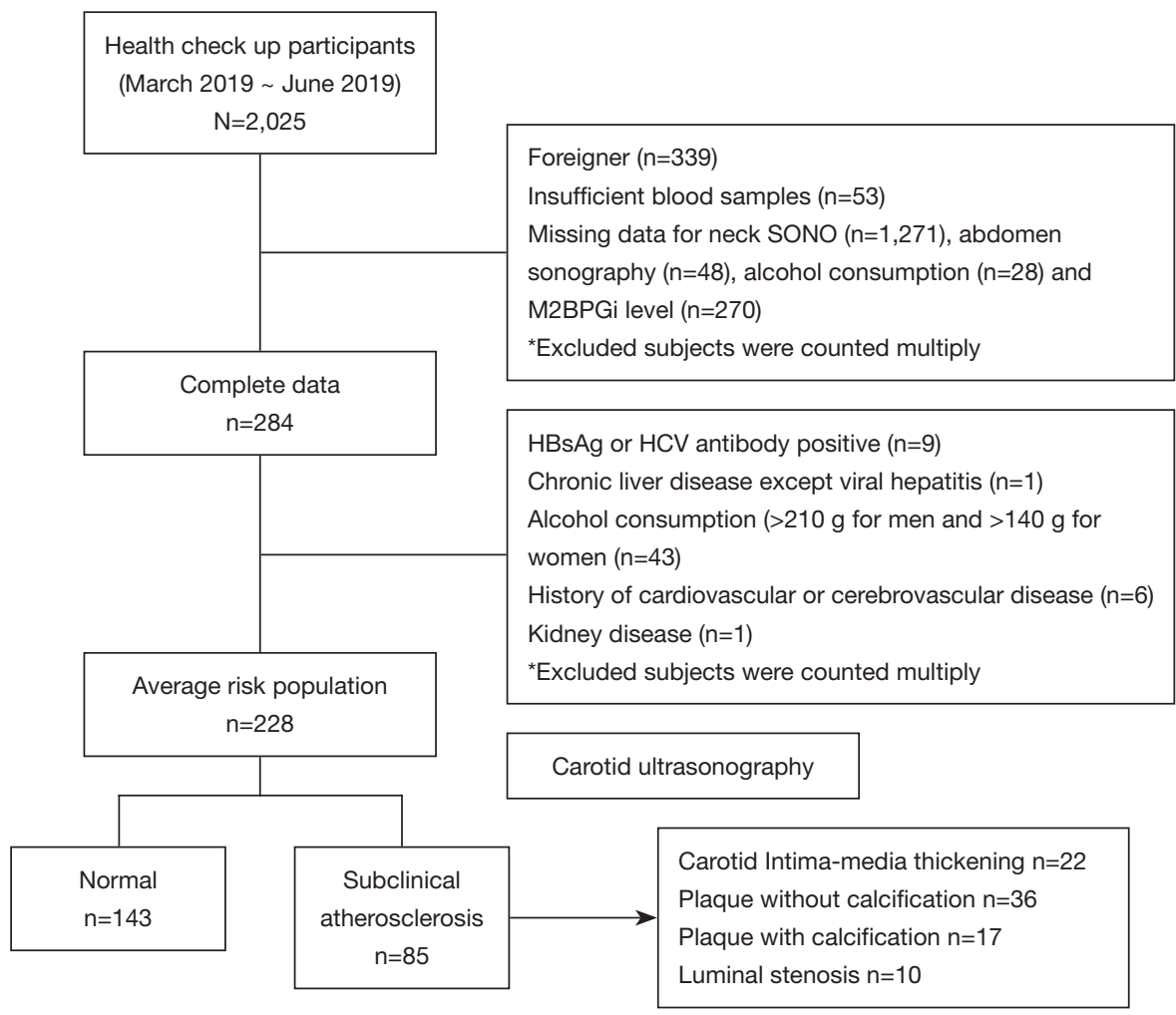

Figure 1 Study flow diagram.

(https://cris.nih.go.kr/cris, Registration No. KCT0004713). The informed consent was waived, because suspected harm to participants was minimal or none.

\section{Inclusion and exclusion criteria}

In this study, a total of 2,025 adult subjects who underwent a health check-up at the International Healthcare Service, Hanyang University Medical Centre, between March 2019 and June 2019 were initially recruited. We excluded foreigner subjects $(\mathrm{n}=339)$ and subjects with insufficient remnant blood samples $(n=53)$ or with missing data for carotid ultrasonography $(\mathrm{n}=1271)$, abdomen sonography $(\mathrm{n}=48)$, alcohol consumption $(\mathrm{n}=28)$ and serum M2BPGi $(\mathrm{n}=270)$. We further excluded subjects from the high-risk liver disease population, those who had positive serologic markers for hepatitis B or hepatitis $\mathrm{C}$ viruses, and those who were self-reported as an HBV carrier $(\mathrm{n}=9)$ or who had chronic liver disease except viral hepatitis $(n=1)$, as well as subjects whose weekly alcohol consumption was greater than $210 \mathrm{~g}$ for men or greater than $140 \mathrm{~g}$ for women $(\mathrm{n}=43)$ and subjects with a virus history of cardiovascular disease $(\mathrm{n}=6)$ or kidney disease $(\mathrm{n}=1)$. Finally, 228 subjects were included as the average-risk population (Figure 1).

\section{Clinical variables, laboratory evaluations and M2BPGi measurement}

Data regarding personal medical and medication histories, smoking and alcohol consumption were collected through a self-reported survey. Smoking and drinking status was categorized as never, past or current smoker/drinker. Bodyweight and height were measured and body mass index (BMI) was calculated using weight in kilograms $(\mathrm{kg})$ divided by height squared $\left(\mathrm{m}^{2}\right)$. Waist circumference (WC) was measured at the narrowest point between the iliac crest and the lower rib margin. Blood pressure $(\mathrm{mmHg})$ was measured at rest in a sitting position.

Serum biochemical variables such as aspartate aminotransferase (AST), alanine aminotransferase (ALT), total cholesterol, triglyceride (TG), high-density lipoprotein (HDL) and low-density lipoprotein cholesterol (LDL), fasting blood sugar (FBS), HbA1c and platelet count (PLT) were measured using conventional methods. 


\section{Page 4 of 11}

Serum M2BPGi quantification was based on a lectin antibody sandwich immunoassay performed using a fully automatic immune-analyzer (HISCL-2000i; Sysmex, Hyogo, Japan) (19). Measurement of serum M2BPGi was supported by Sysmex.

\section{The 10-year ASCVD risk evaluation and definition of bypertension (HTN), diabetes mellitus and byperlipidemia}

ASCVD risk was evaluated using the 10-year ASCVD risk score from the 2013 American College of Cardiology/ American Heart Association (ACC/AHA) guidelines (20). They recommend that statins are prescribed to (I) patients whose LDL $\geq 190 \mathrm{mg} / \mathrm{dL}$, (II) patients with diabetes mellitus (DM) and (III) patients with an estimated 10 -year ASCVD risk $\geq 7.5 \%$ or 40 to 75 years of age who have LDL-C 70 to $189 \mathrm{mg} / \mathrm{dL}$ due to a high cardiovascular disease burden. In this study, a $7.5 \%$ cut-off was used in evaluating diagnostic performance.

The presence of hypertension was defined as systolic blood pressure $\geq 140$, diastolic blood pressure $\geq 90$ or treatment with anti-hypertensive drugs. The presence of $\mathrm{DM}$ was defined as FBS $\geq 126 \mathrm{mg} / \mathrm{dL}, \mathrm{HbA} 1 \mathrm{c} \geq 6.5 \%$ or treatment with anti-diabetic drugs. The presence of hyperlipidemia was defined as LDL $\geq 140$ or treatment with lipid-lowering drugs.

\section{Assessment of atherosclerosis by carotid ultrasonography}

The evaluation of carotid atherosclerosis was based on the Mannheim Carotid Intima-Media Thickness and Plaque Consensus (21). The presence of plaque and maximum plaque thickness, perpendicular length to the carotid longitudinal axis and the presence of calcification was recorded. The severity of atherosclerosis was divided into five stages: normal, carotid intima-media thickness (CIMT) thickening, plaque without calcification, plaque with calcification and luminal stenosis. Luminal stenosis was defined as patients whose carotid artery diameter was narrowed by $25 \%$. Advanced atherosclerosis was defined as subjects with calcified plaque or luminal stenosis (22).

\section{Statistical analysis}

Continuous and categorical variables are presented as mean (standard deviation, SD) and number (per cent), respectively. For comparing the mean values between groups, a t-test or analysis of variance (ANOVA) was

\section{Park et al. M2BPGi and advanced carotid artery atherosclerosis}

used for continuous variables. Categorical variables were compared using chi-square tests. Fisher's exact test was used for categorical variables when $20 \%$ or more of the cells have an expected frequency of less than 5. The Pearson analysis was used for assessing the correlation between M2BPGi level and ASCVD risk.

The diagnostic performance of serum M2BPGi for atherosclerosis was assessed using receiver operator characteristic (ROC) curves. The cut-off value using Youden's index was set at 0.525 to discriminate advanced atherosclerosis (calcified plaque) in the average-risk population.

For all analyses, $\mathrm{P}$ values less than 0.05 were considered as statistically significant. Statistical analyses were performed using SPSS, version 20.0 for Windows (SPSS Inc., Chicago, IL). All subjects who have any missing data were excluded in analysis.

\section{Results}

\section{Characteristics of the study population}

The analysis finally included 228 subjects because 1797 subjects had cardiovascular disease history, risk factors for chronic liver or kidney disease, or insufficient data (Figure 1). The baseline characteristics and demographic data of the 228 study subjects according to the severity of atherosclerosis are presented in Table 1. The mean age of the subjects was 49.11 years, and the proportion of males was $67.1 \%$. The prevalence of subclinical atherosclerosis (CIMT thickening) and advanced atherosclerosis (calcified plaque formation) was $37.2 \%(85 / 228)$ and $11.8 \%(27 / 228)$ in the total subjects. Age, smoking history, fasting glucose, HbA1c and prevalence of hypertension, hyperlipidemia and diabetes significantly increased with severity of atherosclerosis (the trend for all $\mathrm{P}<0.05$ ).

\section{Association between serum M2BPGi and severity of atherosclerosis and 10-year ASCVD risk}

Serum M2BPGi level (0.5712 vs. 0.4913, $\mathrm{P}=0.022)$ and 10 year ASCVD risk $(8.15 \%$ vs. $3.23, \mathrm{P}<0.001)$ were significantly higher in subjects with subclinical atherosclerosis than in the control group (Table 1). Both serum M2BPGi and ASCVD risk increased with the severity of atherosclerosis ( $\mathrm{P}$ for trend $=0.051$ in $\mathrm{M} 2 \mathrm{BPGi}$ and $<0.001$ in ASCVD risk). Serum M2BPGi level was also significantly higher in subjects with calcified plaque and luminal stenosis than those in the control group (Figure 2A). Pearson correlation analysis between 


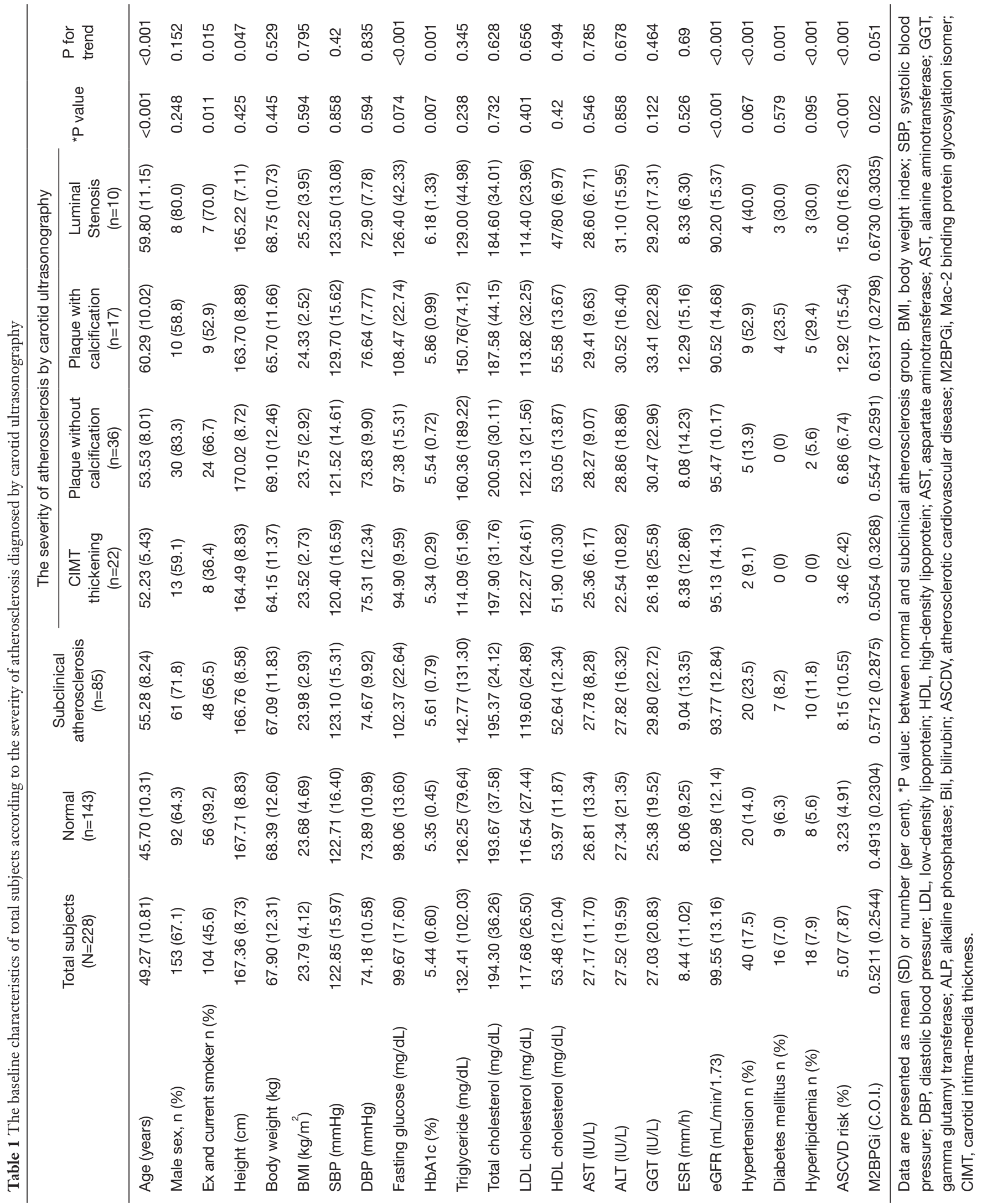



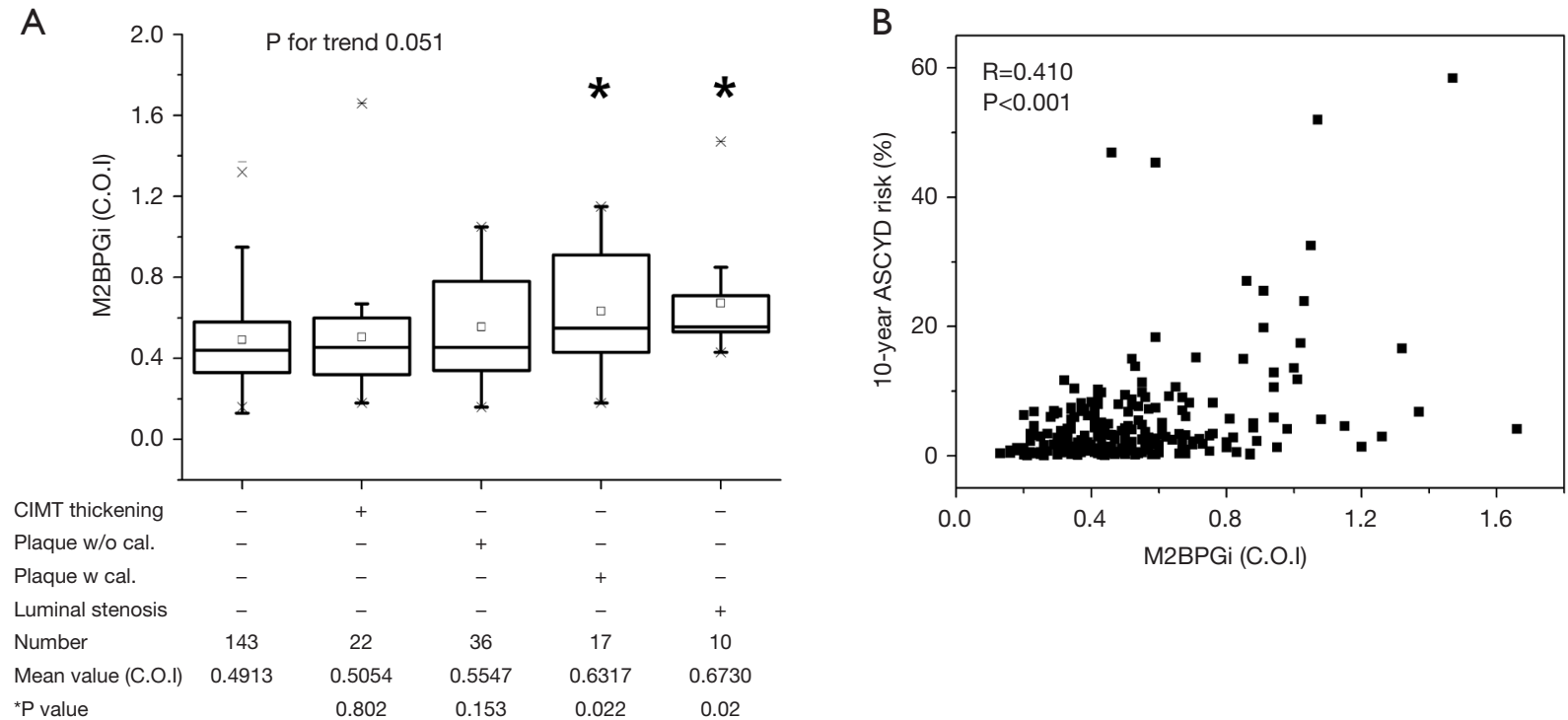

Figure 2 The box plot of M2BPGi versus the severity of subclinical atherosclerosis defined by carotid ultrasonography in an average-risk population (A). The severity of atherosclerosis is divided into five stages: normal, CIMT thickening, plaque without calcification, plaque with calcification and luminal stenosis. The scatter plot serum M2BPGi versus ASCVD risk (B). Asterisk $\left(^{*}\right)$ indicates statistical significance. CIMT, carotid intima-media thickness; cal, calcification; ASCVD, atherosclerotic cardiovascular disease; M2BPGi, Mac-2 binding protein glycosylation isomer.

M2BPGi and ASCVD risk $(\mathrm{R}=0.410, \mathrm{P}<0.001)$ showed a positive relationship (Figure $2 B$ ). All of these results indicate that serum M2BPGi has a positive association with both the severity of atherosclerosis and ASCVD risk.

\section{Diagnostic performance of M2BPGi for identifying the different stages of subclinical atherosclerosis}

Before evaluating the diagnostic performance, the distributions of serum M2BPGi level according to the discriminating stage of atherosclerosis were investigated (Figure $3 A$ ). The severity of atherosclerosis was divided into five stages: normal, CIMT thickening, plaque without calcification, plaque with calcification and luminal stenosis. There was a significant difference in serum M2BPGi level in identifying atherosclerosis stage with CIMT thickening, plaque formation and calcified plaque formation. However, there was no significant difference in serum M2BPGi level in discriminating subjects with luminal stenosis from subjects without luminal stenosis. The ROC curve and area under the receiver operating characteristic (AUROC) used to identify different stages of atherosclerosis are presented in Figure 3B,C, respectively. The AUROCs of serum M2BPGi for identifying $\geq$ CIMT thickening, $\geq$ uncalcified plaque, $\geq$ calcified plaque and $\geq$ luminal stenosis were 0.583 , $0.619,0.679$ and 0.709 , respectively (all $\mathrm{P}<0.05)$.

\section{Comparison of M2BPGi with 10-year ASCVD risk for diagnosing advanced atherosclerosis ( $\geq$ calcified plaque formation)}

When considering the serum M2BPGi level, AUROC and clinical significance, we concluded that identifying the stage above calcified plaque was the most clinically meaningful and effective. Therefore, the diagnostic performance by serum M2BPGi (cut-off 0.525) and 10-year ASCVD risk (cut-off $7.5 \%$ ) to identify advanced atherosclerosis was compared in Figure 4. Sensitivity (66.7\% vs. 51.9\%) and NPV (93.6\% vs. 93.1\%) was higher by M2BPGi than ASCVD risk. However, AUROC (0.679 vs. 0.778), specificity $(65.7 \%$ vs. $87.1 \%)$ and positive predictive value (PPV) (20.7\% vs. 35.0\%) were lower by M2BPGi than by ASCVD risk.

A new sequential algorithm for advanced atherosclerosis in a community-based setting

The performances of ASCVD risk and M2BPGi 
A

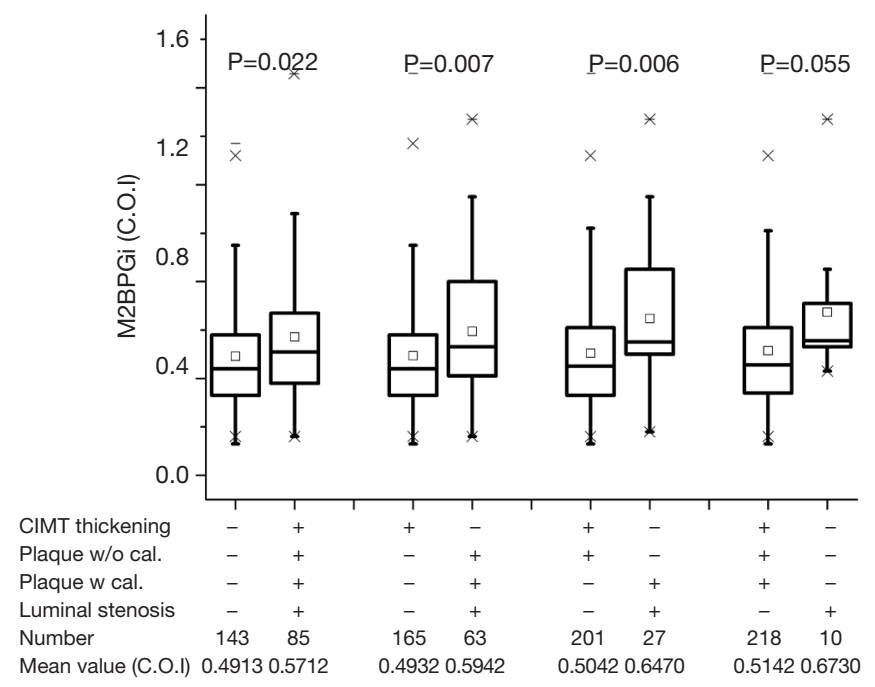

B

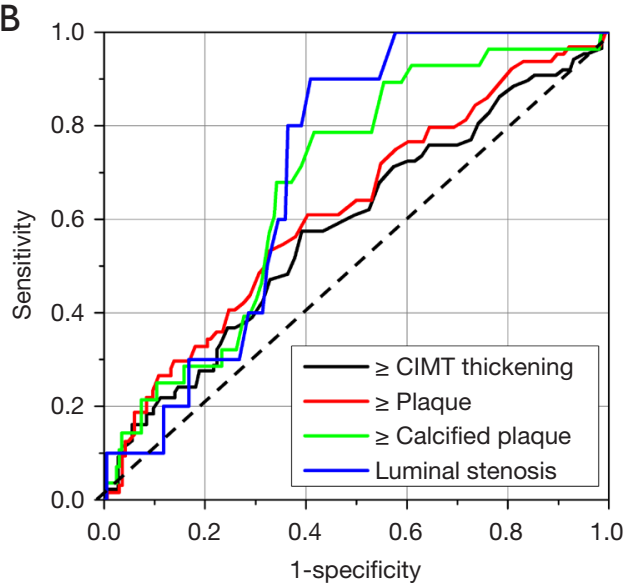

C

\begin{tabular}{|c|c|c|}
\hline & AUROC & P value \\
\hline CIMT thickening & 0.583 & 0.035 \\
\hline Plaque & 0.619 & 0.005 \\
\hline Calcified plaque & 0.679 & 0.003 \\
\hline Luminal stenosis & 0.709 & 0.025 \\
\hline
\end{tabular}

Figure 3 The box plot of M2BPGi versus the status of atherosclerosis defined by carotid ultrasonography (A). Receiver operating characteristic (ROC) curves for diagnosis of $\geq$ CIMT thickening (black line), $\geq$ plaque formation (red line), $\geq$ calcified plaque formation (green line), and presence of luminal stenosis (blue line) by M2BPGi (B). Their AUROCs were summarized in (C). AUROC, area under the receiver operating characteristic; CIMT, carotid intima-media thickness.

A

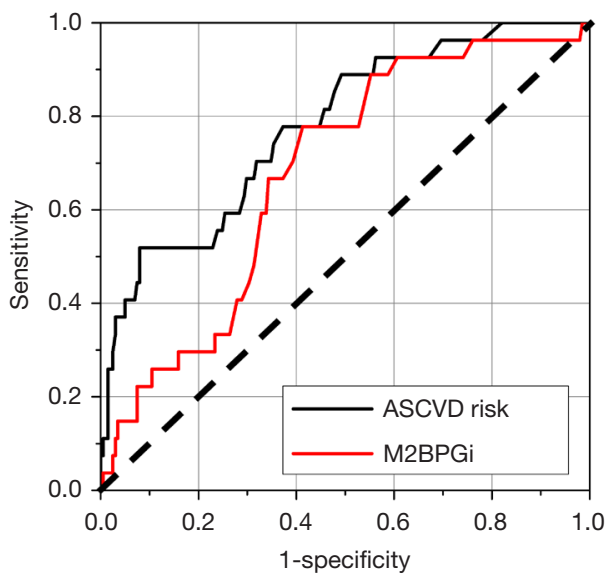

B

\begin{tabular}{|c|c|c|c|c|c|c|}
\hline & AUROC & P value & Sensitivity (\%) & Specificity (\%) & PPV (\%) & NPV (\%) \\
\hline ASCVD risk & 0.778 & $<0.001$ & 51.9 & 87.1 & 35.0 & 93.1 \\
\hline M2BPGi & 0.679 & 0.003 & 66.7 & 65.7 & 20.7 & 93.6 \\
\hline
\end{tabular}

Figure 4 ROC curves for diagnosis of advanced atherosclerosis ( $\geq$ calcified plaque formation) by ASCVD risk (black line), and serum M2BPGi (red line) (A). Summary of diagnostic performance (B). ROC, receiver operating characteristic; AUROC, area under the receiver operating characteristic; ASCVD, atherosclerotic cardiovascular disease; M2BPGi, Mac-2 binding protein glycosylation isomer; PPV, positive predictive value; NPV, negative predictive value. 


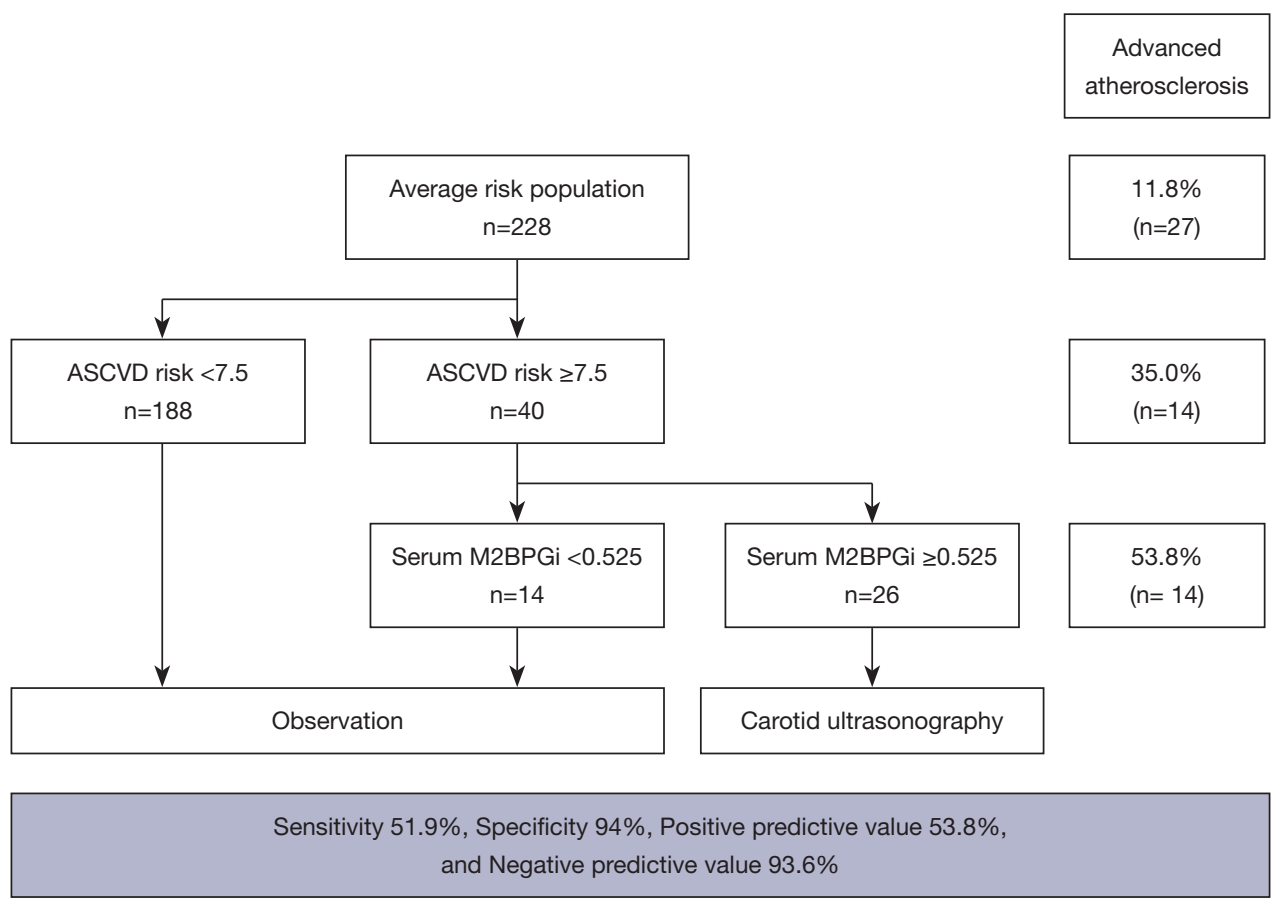

Figure 5 A sequential algorithm for advanced atherosclerosis diagnosis. ASCVD risk evaluation was incorporated following serum M2BPGi. ASCVD, atherosclerotic cardiovascular disease; M2BPGi, Mac-2 binding protein glycosylation isomer.

individually were not accurate enough to predict advanced atherosclerosis as a single indicator. We proposed a new sequential algorithm, the incorporation of ASCVD risk evaluation following serum M2BPGi analysis, to increase the diagnostic performance and avoid unnecessary examination (Figure 5). The sensitivity, specificity, PPV and NPV of the sequential algorithm for advanced atherosclerosis were $51.9 \%, 94.0 \%, 53.8 \%$ and $93.6 \%$, respectively. When the sequential algorithm was used, PPV increased from $35.0 \%$ to $53.8 \%$ without reducing sensitivity, compared to the use of ASCVD risk ( $\geq 7.5 \%)$ alone.

\section{Avoidance of unnecessary carotid ultrasonography by the use of the sequential algorithm}

The avoidance rate of unnecessary carotid ultrasonography by using the sequential algorithm was evaluated. First, 40 subjects were classified into an intermediate-risk group by ASCVD risk ( $\geq 7.5 \%)$. Then, 14 subjects among the intermediate-risk group were screened by M2BPGi as observation group (Figure 5). Interestingly, there were no subjects with advanced atherosclerosis among the subjects screened by M2BPGi. These findings mean that 35.0\%
(14/40) of intermediate-risk subjects determined by ASCVD risk $(\geq 7.5 \%)$ could avoid unnecessary examinations.

\section{Discussion}

In this study, the positive association between serum M2BPGi and the severity of subclinical atherosclerosis and 10-year ASCVD risk were observed in a communitybased setting. When diagnostic performance for identifying advanced atherosclerosis was evaluated, sequential algorithm showed better performance than method by ASCVD or M2BPGi alone.

Many carotid sonography examinations are performed for uncertain indications in the real-world setting. Most guidelines recommend that carotid sonography is performed only in limited cases. However, there are conflicting recommendations among guidelines. Pooled cohort equations to predict the 10-year risk for ASCVD did not correlate satisfactorily with advanced imaging features of carotid artery atherosclerosis. A sequential algorithm using ASCVD $(\geq 7.5)$ and M2BPGi $(\geq 0.525)$ is a reasonable indication for carotid artery sonography in a community-based setting.

We adopted a sequential algorithm to select a target population for carotid artery sonography. ASCVD was 
placed as the first screening step for the algorithm because ASCVD is one of the best validated indexes for stratifying risk groups. We tried to avoid carotid artery sonography in the very low-risk population. Then, we used M2BPGi as the second screening step because the ASCVD risk score did not predict advanced atherosclerosis. Among patients with an ASCVD score of $\geq 7.5 \%$, only $45.3 \%$ of subjects had carotid artery soft plaque and $63.8 \%$ had carotid arterial plaque (10). Only $46.4 \%$ of patients with a 10 -year ASCVD score of $7.5 \%$ or greater had coronary artery stenosis (23). These findings were similar with our results. Prevalence of carotid atherosclerosis and advanced carotid atherosclerosis in subjects with intermediate-risk (ASCVD risk $\geq 7.5$ ) was only $60.0 \%$ and $35.0 \%$, respectively, in our data. In other words, $40 \%$ of subjects categorized as intermediaterisk had normal carotid arteries. Moreover, the diagnostic performance by ASCVD risk could not predict the severity of atherosclerosis effectively. This is the reason why we used M2BPGi after the ASCVD risk score. As a result, 35.0\% $(14 / 40)$ of the intermediate-risk subjects determined by ASCVD risk ( $\geq 7.5 \%$ ) could avoid unnecessary examinations. These results originated from high NPV $(93.6 \%)$ of serum M2BPGi.

Originally, serum M2BPGi was developed to predict fibrosis of the liver (24). However, serum M2BPGi has a common feature related with the activation of macrophages in the disease progression of liver fibrosis and atherosclerosis. Serum M2BPGi acts as a juxtacrine-signalling messenger sent by hepatic stellate cells to Kupffer cells during the progression of liver fibrosis $(11,25)$. Serum M2BPGi helps circulating monocytes bind to adhesion molecules expressed by activated endothelial cells during the progression of atherosclerosis $(14,15,18)$. Serum M2BPGi does not represent total fibrosis or atherosclerosis but disease activity. Therefore, after effective antiviral therapy (11) or CVD attack (26), serum M2BPGi level decreases according to disease activity. Although it is only our hypothesis, these results are evidence that both liver fibrosis and atherosclerosis have a common ground in pathophysiology and M2BPGi can be used as a diagnostic marker not only for liver fibrosis but also for atherosclerosis. However, this feature can result in a decrease in specificity. It is important to consider the effect from liver fibrosis, when evaluating the degree of atherosclerosis by using M2BPGi.

This study has several limitations. First, the sample size was small. In particular, there were only ten subjects with luminal stenosis. Second, the causal relationship between serum M2BPGi and severity of atherosclerosis and ASCVD risk cannot be discussed due to the study design. Therefore, large-scale longitudinal studies will be needed for a more accurate analysis. We have the plan to do longitudinal study to know the association between serum M2BPGi and prevalence of cardiovascular disease such as stroke, myocardial infarct. Third, further study is needed to confirm our hypothesis about the common pathophysiology between liver fibrosis and atherosclerosis. Finally, clinical validity will be needed to use our sequential diagnostic flow for screening advanced atherosclerosis.

Regardless of these limitations, this study has some strengths. First, this study is the first study to show the association between M2BPGi and the severity of atherosclerosis by using carotid imaging method. Second, this study suggests reasonable indication for carotid artery imaging test. Though most guidelines recommend not to use carotid artery imaging in the general population, because their imperfect sensitivity could lead unnecessary surgery or intervention which could result in serious harms such as stroke (6), many carotid sonography examinations are performed for uncertain indications in the real-world setting. Moreover, other confirmed tools do not exist except cardiovascular risk assessment method, which is not enough to be used as a single indicator. In this situation, new sequential algorithm to increase the performance of risk estimator will be reasonable screening tool. In addition, the low cost and simple procedure of M2BPGi analysis make sequential algorithm to be easily applied for screening advanced atherosclerosis especially in primary clinical or health check-up settings.

In conclusion, our results showed that serum M2BPGi has a positive association with the severity of atherosclerosis established by carotid ultrasonography and 10-year ASCVD risk. When the sequential algorithm of ASCVD risk evaluation followed by serum M2BPGi was applied, PPV was increased from $35.0 \%$ to $53.8 \%$ without reducing sensitivity. As a result, $35.0 \%(14 / 40)$ of intermediate-risk subjects by ASCVD risk $(\geq 7.5 \%)$ could avoid unnecessary examination. The sequential algorithm can be used for screening advanced atherosclerosis and indication for carotid ultrasound in a community-based setting.

\section{Acknowledgments}

Funding: This study was supported by Sysmex. The funding source had no role in the study design, implementation, data collection, analysis, interpretation or in the preparation, review or approval of the manuscript. 


\section{Footnote}

Reporting Checklist: The authors have completed the STROBE reporting checklist. Available at http://dx.doi. org/10.21037/atm-20-7219

Data Sharing Statement: Available at http://dx.doi. org/10.21037/atm-20-7219

Conflicts of Interest: All authors have completed the ICMJE uniform disclosure form (available at http://dx.doi. org/10.21037/atm-20-7219). All authors have no competing or conflicts of interest to declare.

Ethical Statement: The authors are accountable for all aspects of the work in ensuring that questions related to the accuracy or integrity of any part of the work are appropriately investigated and resolved. The study was conducted in accordance with the Declaration of Helsinki (as revised in 2013). The Institutional Review Board (IRB) of Hanyang University Medical Centre approved this study protocol (IRB No. HYUH 2020-01-035). The protocol was also registered at the Clinical Research Information Service (https://cris.nih.go.kr/cris, Registration No. KCT0004713). The informed consent was waived, because suspected harm to participants was minimal or none.

Open Access Statement: This is an Open Access article distributed in accordance with the Creative Commons Attribution-NonCommercial-NoDerivs 4.0 International License (CC BY-NC-ND 4.0), which permits the noncommercial replication and distribution of the article with the strict proviso that no changes or edits are made and the original work is properly cited (including links to both the formal publication through the relevant DOI and the license). See: https://creativecommons.org/licenses/by-nc-nd/4.0/.

\section{References}

1. Herrington W, Lacey B, Sherliker P, et al. Epidemiology of Atherosclerosis and the Potential to Reduce the Global Burden of Atherothrombotic Disease. Circ Res 2016;118:535-46.

2. Libby P, Buring JE, Badimon L, et al. Atherosclerosis. Nat Rev Dis Primers 2019;5:56.

3. Shaw LJ, Taylor A, Raggi P, et al. Role of noninvasive imaging in asymptomatic high-risk patients. J Nucl Cardiol 2006;13:156-62.
4. Toth PP. Subclinical atherosclerosis: what it is, what it means and what we can do about it. Int J Clin Pract 2008;62:1246-54.

5. Iwasaki K, Matsumoto T, Aono H, et al. Prevalence of subclinical atherosclerosis in asymptomatic patients with low-to-intermediate risk by 64-slice computed tomography. Coron Artery Dis 2011;22:18-25.

6. US Preventive Services Task Force. Final Update Summary. Carotid artery stenosis: screening. Jul. 2014. Accessed December 1, 2015. Available online: http:// www.uspreventiveservicestaskforce.org/Page/Document/ UpdateSummaryFinal/carotidartery-stenosis-screening

7. Andrus B, Lacaille D. 2013 ACC/AHA guideline on the assessment of cardiovascular risk. J Am Coll Cardiol 2014;63:2886.

8. AIUM Practice Parameter for the Performance of an Ultrasound Examination of the Extracranial Cerebrovascular System. Laurel, MD: American Institute of Ultrasound in Medicine; 2011. Accessed November 18, 2015. Available online: http://www.aium.org/resources/ guidelines/extracranial.pdf.

9. Brott TG, Halperin JL, Abbara S, et al. 2011 ASA/ACCF/ AHA/AANN/AANS/ACR/ASNR/CNS/SAIP/SCAI/SIR/ SNIS/SVM/SVS guideline on the management of patients with extracranial carotid and vertebral artery disease: executive summary. Stroke 2011;42:e420-63.

10. Li Y, Zhu G, Ding V, et al. Assessing the Relationship between Atherosclerotic Cardiovascular Disease Risk Score and Carotid Artery Imaging Findings. J Neuroimaging 2019;29:119-25.

11. Shirabe K, Bekki Y, Gantumur D, et al. Mac-2 binding protein glycan isomer (M2BPGi) is a new serum biomarker for assessing liver fibrosis: more than a biomarker of liver fibrosis. J Gastroenterol 2018;53:819-26.

12. Cho SY, Hur M. Mac-2 Binding Protein Glycosylation Isomer: Emerging Non-Invasive Serum Marker for Liver Fibrosis. Ann Lab Med 2018;38:289-90.

13. Koopmann J, Thuluvath PJ, Zahurak ML, et al. Mac-2binding protein is a diagnostic marker for biliary tract carcinoma. Cancer 2004;101:1609-15.

14. Shirure VS, Reynolds NM, Burdick MM. Mac-2 binding protein is a novel E-selectin ligand expressed by breast cancer cells. PLoS One. 2012;7:e44529.

15. Sasaki T, Brakebusch C, Engel J, et al. Mac-2 binding protein is a cell-adhesive protein of the extracellular matrix which self-assembles into ring-like structures and binds $\beta 1$ integrins, collagens and fibronectin. EMBO J 1998;17:1606-13. 
16. Niinaga R, Yamamoto H, Yoshii M, et al. Marked elevation of serum M2BP-adiponectin complex in men with coronary artery disease. Atherosclerosis 2016;253:70-4.

17. Sugiura T, Dohi Y, Takase H, et al. Serum levels of Mac-2 binding protein increase with cardiovascular risk and reflect silent atherosclerosis. Atherosclerosis 2016;251:192-6.

18. Sugiura T, Dohi Y, Takase H, et al. Factors associated with longitudinal changes in serum concentrations of Mac-2 binding protein: A prospective 3-year observational study. Nutr Metab Cardiovasc Dis 2019;29:1337-44.

19. Kuno A, Ikehara Y, Tanaka Y, et al. A serum "sweetdoughnut" protein facilitates fibrosis evaluation and therapy assessment in patients with viral hepatitis. Sci Rep 2013;3:1065.

20. Goff DC Jr, Lloyd-Jones DM, Bennett G, et al. 2013 ACC/AHA guideline on the assessment of cardiovascular risk: a report of the American College of Cardiology/ American Heart Association Task Force on Practice Guidelines. Circulation 2014;129:S49-73.

21. Touboul PJ, Hennerici MG, Meairs S, et al. Mannheim carotid intima-media thickness and plaque consensus (2004-2006-2011). An update on behalf of the advisory board of the $3 \mathrm{rd}$, 4th and 5 th watching the risk symposia, at the 13th, 15th and 20th European Stroke Conferences,

Cite this article as: Park H, Jun DW, Park HK, Park KY, Hwang HS. New sequential algorithm using Mac-2 binding protein glycosylation isomer to detect advanced carotid artery atherosclerosis. Ann Transl Med 2021;9(7):566. doi: 10.21037/ atm-20-7219
Mannheim, Germany, 2004, Brussels, Belgium, 2006, and Hamburg, Germany, 2011. Cerebrovasc Dis 2012;34:290-6.

22. Stary HC, Chandler AB, Dinsmore RE, et al. A definition of advanced types of atherosclerotic lesions and a histological classification of atherosclerosis. A report from the Committee on Vascular Lesions of the Council on Arteriosclerosis, American Heart Association. Circulation 1995;92:1355-74.

23. Li Y, Zhu G, Ding V, et al. Assessing the Relationship Between American Heart Association Atherosclerotic Cardiovascular Disease Risk Score and Coronary Artery Imaging Findings. J Comput Assist Tomogr 2018;42:898-905.

24. Kim M, Jun DW, Park H, et al. Sequential Combination of FIB-4 Followed by M2BPGi Enhanced Diagnostic Performance :for Advanced Hepatic Fibrosis in an Average Risk Population. J Clin Med 2020;9:1119.

25. Bekki Y, Yoshizumi T, Shimoda S, et al. Hepatic stellate cells secreting WFA+-M2BP: its role in biological interactions with Kupffer cells. J Gastroenterol Hepatol 2017;32:1387-93.

26. Xie H, Chen L, Liu H, et al. Expression of Mac-2 binding protein in human carotid atheroma is associated with plaque instability and clinical manifestations. Biomed Pharmacother 2019;110:465-72. 